\title{
Acoustic Change Complex: Clinical Implications
}

\author{
Jae-Ryong Kim \\ Department of Otolaryngology-Head and Neck Surgery, Busan Paik Hospital, Inje University College of Medicine, Busan, Korea
}

Received November 12, 2015

Revised November 16, 2015

Accepted November 18, 2015

\author{
Address for correspondence \\ Jae-Ryong Kim, MD, PhD \\ Department of Otolaryngology- \\ Head and Neck Surgery, \\ Busan Paik Hospital, Inje University \\ College of Medicine, 75 Bokji-ro, \\ Busanjin-gu, Busan 47392, Korea \\ Tel +82-51-890-6379 \\ Fax +82-51-892-3831 \\ E-mail jrkim53@hanmail.net
}

\begin{abstract}
The acoustic change complex (ACC) is a cortical auditory evoked potential elicited in response to a change in an ongoing sound. The characteristics and potential clinical implications of the $\mathrm{ACC}$ are reviewed in this article. The P1-N1-P2 recorded from the auditory cortex following presentation of an acoustic stimulus is believed to reflect the neural encoding of a sound signal, but this provides no information regarding sound discrimination. However, the neural processing underlying behavioral discrimination capacity can be measured by modifying the traditional methodology for recording the P1-N1-P2. When obtained in response to an acoustic change within an ongoing sound, the resulting waveform is referred to as the ACC. When elicited, the ACC indicates that the brain has detected changes within a sound and the patient has the neural capacity to discriminate the sounds. In fact, results of several studies have shown that the ACC amplitude increases with increasing magnitude of acoustic changes in intensity, spectrum, and gap duration. In addition, the ACC can be reliably recorded with good test-retest reliability not only from listeners with normal hearing but also from individuals with hearing loss, hearing aids, and cochlear implants. The ACC can be obtained even in the absence of attention, and requires relatively few stimulus presentations to record a response with a good signal-to-noise ratio. Most importantly, the ACC shows reasonable agreement with behavioral measures. Therefore, these findings suggest that the ACC might represent a promising tool for the objective clinical evaluation of auditory discrimination and/or speech perception capacity.

J Audiol Otol 2015;19(3):120-124
\end{abstract}

KEY WORDS: Cortical auditory evoked potential · Acoustic change complex Auditory discrimination.

\section{Introduction}

Cortical auditory evoked potentials (CAEPs) are brain responses evoked by sound and are processed in or near the auditory cortex [1]. To date, a number of CAEPs have been described in the literature. There has been considerable clinical and scientific interest in CAEPs to probe threshold and suprathreshold auditory processes because they are believed to reflect the neural detection and/or discrimination of sound underlying speech perception. These measures include obligatory evoked potentials such as $\mathrm{P} 1, \mathrm{~N} 1$, and $\mathrm{P} 2$, and discriminative potentials such as mismatch negativity (MMN) and P300.

This is an Open Access article distributed under the terms of the Creative Commons Attribution Non-Commercial License (http://creativecommons. org/licenses/by-nc/3.0/) which permits unrestricted non-commercial use, distribution, and reproduction in any medium, provided the original work is properly cited.
The P1-N1-P2 is a transient auditory evoked potential that can be recorded from surface electrodes placed on the scalp in response to a wide range of stimuli, although this potential is typically evoked by a brief stimulus such as clicks, tone bursts, and short duration speech tokens. This obligatory cortical potential consists of three peaks that are recorded within a latency range extending from 50 to $200 \mathrm{~ms}$. The peaks are traditionally labeled individually as $\mathrm{P} 1, \mathrm{~N} 1$, and $\mathrm{P} 2$. The $\mathrm{P} 1-$ N1-P2 recorded from the auditory cortex following presentation of an acoustic stimulus is believed to reflect the neural encoding of a sound signal, but this provides no information on sound discrimination [1-3]. However, the neural processing underlying behavioral discrimination capacity can be measured by modifying the traditional methodology for recording the P1-N1-P2. When obtained in response to an acoustic change within a sound or in response to a stimulus that contains multiple time-varying acoustic changes such as 
speech, the resulting waveform has been referred to as the acoustic change complex (ACC) [2].

Traditionally, MMN and P300 have been used to assess sound discrimination. MMN was discovered by Näätänens, et al. [4] in 1978. It is evoked by an oddball paradigm, in which infrequent deviant sounds are embedded in a series of frequent standard sounds. MMN provides an index of the preattentive discrimination of two or more sounds. Because the MMN is obtained during passive listening, it may be used to index sound discrimination abilities in those who are difficult to test with conventional methods. However, this potential has recognized limitations. MMN has a small wave amplitude, imprecise latency calculations, and relatively poor reliability [2,5]. P300 was first described by Sutton, et al. [6] in 1965. It occurs at approximately $300 \mathrm{~ms}$. This is best evoked when the subject is engaged in a discrimination task, using an oddball paradigm. Subjects are instructed to count in response to a deviant or target stimulus embedded in a train of frequent standard stimuli. This may be more useful for clinical assessment of sound discrimination and cognitive processing. Nonetheless, this potential is difficult to record in uncooperative patients because it requires active participation [7].

Due to the limitation of MMN and P300, the ACC has drawn considerable attention as another method of investigating auditory discrimination. This article provides an overview of recent ACC studies with an emphasis on the characteristics and potential implications for clinicians and audiologists.

\section{The ACC Indicates the Encoding of Potentially Discriminable Information at the Level of the Auditory Cortex}

The ACC is a CAEP evoked in response to a change in an ongoing sound [2]. Ostroff, et al. [8] recorded cortical potentials in response to three naturally produced speech stimuli (/s/, /ei/, and /sei/) in adults with normal hearing. They found that the response evoked by the speech syllable /sei/ consisted of two overlapping onset responses generated by the onsets of the sibilant/s/ and the vowel/ei/, respectively. Therefore, they suggested that this potential to multiple time-varying speech waveforms can reflect a change in the acoustic characteristics of speech signals within a syllable. In a companion study, Martin and Boothroyd [9] measured responses to changes in stimulus level and/or spectral content of long-duration, ongoing stimuli. Using the synthetic vowel $/ \mathrm{u} /$ to elicit cortical responses, they introduced a change to the intensity and/or frequency of the second formant frequency (from $/ \mathrm{u} /$ to $/ \mathrm{i} /$ ). The ACC amplitude increased with increasing magnitude of acoustic change.
In addition, several studies have shown that the ACC response can be recorded using ongoing stimuli that contain silent gaps of various durations [10-13]. Lister, et al. [12] used narrowband noise bursts centered at 2000 or $1000 \mathrm{~Hz}$ with a temporal gap introduced into the burst to elicit a change response. They observed larger amplitude responses when the gap duration was increased.

Overall, the results of these studies indicate that the ACC can be reliably elicited by changes in intensity [9,14-16], spectrum [15-17], and/or gap duration [10,12]. In addition, these studies showed that the ACC varies systematically depending on the specific acoustic characteristics of the sound. Therefore, the ACC might serve as an objective measure of the neural processes that underlie the changes in an ongoing acoustic stimulus. Consequently, it has been suggested that the ACC might indicate the encoding of potentially discriminable information at the level of the auditory cortex $[2,8,9]$.

\section{The ACC May Serve as a Potentially Useful Measure for the Clinical Assessment of Speech Perception Capacity}

Several prerequisites are needed for the ACC to serve as a clinically useful tool for assessing speech perception ability. A summary of several studies that have provided evidence that the ACC satisfies some of these prerequisites follows.

\section{The ACC shows excellent test-retest reliability}

First, if CAEPs such as the ACC are to hold promise as clinical tools for assessing sound discrimination, it is necessary to determine whether various stimuli evoke distinct neural patterns and whether the neural responses are reliable in individual participants. The reliability of the ACC has been reported in a number of studies [18-20]. Tremblay, et al. [18] used four naturally produced speech tokens (/bi/, /pi/, / $\mathrm{ji} /$, and $/ \mathrm{si} /$ ) to measure the neural detection of acoustic cues in seven listeners with normal hearing. The ACC response evoked by the /bi/ stimulus had a larger amplitude than the response elicited by /pi/, and the response to / $\mathrm{j} /$ had a shorter latency than the response to /si/. That is, the stimuli, representing different acoustic cues, evoked distinct neural response patterns. In addition, the ACC responses for each stimulus obtained from individuals were stable across trials. Thus, these results indicate that the ACC shows remarkable test-retest reliability.

\section{The ACC can be recorded in children}

The need for objective measures in children with hearing 
loss has recently increased due to hearing aid use and cochlear implantation at younger ages. Therefore, it is important to be able to measure the ACC, which is assumed to reflect auditory discrimination, in infants and other children and use it to establish guidelines for both intervention and for follow-up outcomes. Although the results have been preliminary, some studies have investigated whether the ACC can be recorded in infants and children [21,22]. Martinez, et al. [21] showed that the ACC for /u/-/i/ (vowel place contrast) or / $\mathrm{u} /$-/a/ (vowel height contrast) was successfully recorded in five children with normal hearing and five with hearing loss, suggesting that the ACC can be recorded reliably in children.

The ACC can be elicited in individuals with hearing aids and cochlear implants

To date, several studies have shown that the ACC can also be recorded in individuals with hearing aids (HAs) and cochlear implants (CIs). Tremblay, et al. [19] examined the effects of amplification on the reliability of the ACC. Naturally produced speech stimuli (/si/ and $/ \mathrm{ji} /$ ) were used to evoke the ACC in both unaided and aided conditions. The results indicated that the ACC can be recorded reliably in individuals with HAs, and the differences in the ACC between /si/ and / $\int \mathrm{i} /$ were maintained in the aided condition.

Friesen and Tremblay [20] were the first to report measuring the electrically evoked ACC (EACC) in CI users. They used two stimuli, /si/ and $/ \mathrm{Ji} /$, presented in the sound field and recorded the EACC. Their study showed that, despite the stimulus artifact associated with electrical stimulation, the
EACC could be reliably recorded in CI users and that different stimuli evoked distinct waveforms. In addition, several other studies have used different stimuli presented either through a speech processor or directly to individual electrodes to elicit the EACC in CI users [23-25]. Brown, et al. [24] and Kim, et al. [25] used long-duration, biphasic pulse trains as stimuli instead of a speech stimulus presented in the sound field, and showed that CI output was controlled directly rather than through the speech processor. The EACC was recorded in response to changes in the stimulating electrode or at the level of stimulation on one electrode. The amplitude of the EACC was dependent on the magnitude of the stimulus change. Fig. 1 shows examples of the EACC evoked by changes in spectra, time, and intensity. In this example, the amplitudes of the EACC increase as a function of changes in the stimulus.

\section{The ACC has an advantage over the MMN}

The ACC is similar to the MMN, which reflects discrimination capacity in the absence of attention. However, the ACC has a much larger amplitude (higher signal-to-noise ratio) and requires fewer stimulus presentations because every trial contributes to the response [2]. Therefore, this may be an advantage of ACC over the MMN [10].

\section{The ACC shows good agreement with behavioral measures}

A number of studies have suggested that the ACC may be a potentially useful measure in clinical assessment of speech
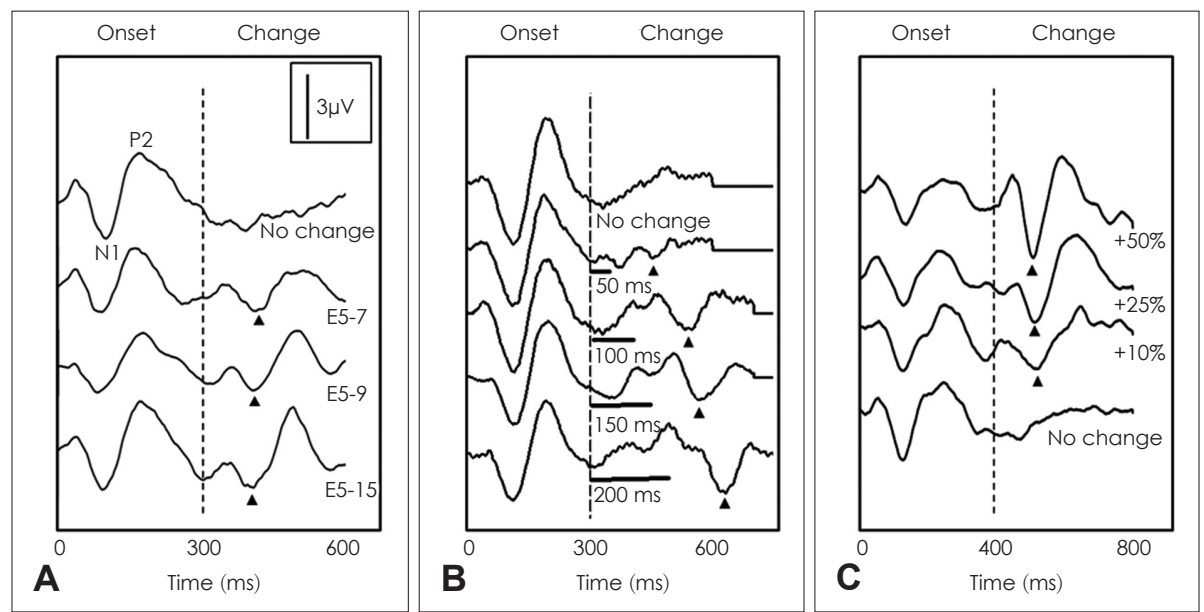

Fig. 1. Examples of onset and electrically evoked acoustic change complex (EACC) responses recorded from implanted users according to changes in spectra, time, and intensity. The EACC amplitudes have a tendency to increase as a function of changes in the stimulus. A: EACC recordings where the stimulus is a change in the stimulation electrode. In each case, stimulation began on electrode 5 . At $300 \mathrm{~ms}$ after stimulus onset, the stimulation was changed to a different electrode. The stimulated electrode for the second segment is indicated. B: EACC recordings evoked by changing the gap duration. In the gap condition, a temporal gap (silent interval) was inserted after $300 \mathrm{~ms}$ of stimulation. Gap duration is indicated. C: EACC recordings elicited by a change in stimulus level. In each case, the stimulus was initially presented at $50 \%$ of the subject's dynamic range. The current level used for the second 400 ms of the stimulation was varied. The percent increment in level is indicated. In each panel, dashed vertical lines indicate the onset of stimulus change. The small triangle indicates the point in the waveform that was identified as the N1 of EACC. 
perception. However, most of all, this assumption is based on the observations of good agreement between the ACC and behavioral measures of auditory discrimination.

To date, several studies have investigated the relationship between the ACC and behavioral measures of auditory discrimination. Martin and Boothroyd [9] found that the ACC thresholds evoked by an intensity change compared favorably with those obtained from other psychophysical studies. He et al. [26] systematically investigated the relationship between the ACC and behavioral measures of auditory discrimination across various stimulus dimensions (temporal, spectral, and intensity dimension) in individuals with normal hearing. They individually compared psychophysical thresholds for gap detection, frequency discrimination, and intensity discrimination with ACC thresholds. They found that the ACC and behavioral measures for frequency and intensity discrimination were significantly correlated.

Some researchers have also examined the relationship between the ACC and behavioral measures in individuals with auditory neuropathy spectrum disorder (ANSD) [10,16,27]. ANSD is characterized by an absent or severely abnormal auditory brainstem response with preservation of cochlear microphonics and/or otoacoustic emissions [28]. This finding indicates that outer hair cells remain intact, but the function of inner hair cells or auditory nerves is impaired. A common pathophysiological mechanism of ANSD is compromised neural synchrony, which leads to temporal processing deficits [29]. In other words, ANSD is a hearing disorder that affects the processing of auditory temporal cues that are essential for understanding speech. Michalewski, et al. [10] examined the ACC evoked by gaps in noise to assess temporal processing in both normal hearing and ASND individuals. Their results revealed that the ACC appeared with a more prolonged gap duration in the ANSD group than in those with normal hearing. In addition, they revealed a close association between gap detection thresholds measured psychophysically and electrophysiologically in both the normal and ANSD groups. He, et al. [27] also recorded the ACC evoked by temporal gaps in children with ANSD. They investigated the relationship between gap detection thresholds measured by the ACC and speech perception in these subjects. Their results showed that objective gap detection thresholds were significantly correlated with aided PBK word scores. These data indicate that subjects with prolonged objective gap detection thresholds show poorer speech performance. Therefore, the authors suggested that the ACC can be used as an objective tool to identify poor performers and potential candidates for CI in children with ANSD.

CI has become the treatment of choice for severe to pro- found hearing loss. Although remarkable speech understanding has been obtained in CI users, there is still large variability with regard to outcomes. Therefore, several researchers have attempted to examine the relationship between objective measures and speech performance for predicting outcome and aiding in auditory rehabilitation, particularly in young, difficult-to-test children. Won, et al. [30] examined the relationship between behavioral and physiologic measures for spectral resolution in listeners with normal hearing. There was a robust correlation between ACC amplitude and behavioral spectral ripple discrimination. Thus far, several studies have demonstrated that behavioral spectral ripple discrimination ability is significantly correlated with speech perception in CI users [31,32]. Therefore, this study indirectly showed that the ACC may be used to assess spectral resolution, even in CI users. He, et al. [33,34] reported the relationship between the EACC and speech perception in ANSD children with CI. They used the stimulation paradigm originally described by Brown, et al. [24]. In the first study [33], they recorded the EACC in response to temporal gaps. They reported a robust negative correlation between the EACC thresholds for gap detection and Phonetically Balanced Kindergarten (PBK) word scores. That is, implanted ANSD children with poorer speech perception showed higher EACC thresholds. In the other study [34], the EACC was evoked by a change in the stimulating electrode. This study also showed a robust correlation between EACC thresholds for electrode discrimination and PBK word scores. In other words, subjects with poorer speech perception required a larger separation between the stimulating electrodes to evoke the EACC. These findings suggest that the EACC, which is elicited by changes in temporal gaps and/or the stimulating electrode, may serve as a very promising tool for objective evaluation of temporal and/or spectral resolution, as well as for predicting outcomes among implanted children with ANSD.

\section{Conclusion}

A number of studies have provided evidence that the ACC, as a CAEP in response to a change in an ongoing sound, indicates the encoding of discriminable information at the level of the auditory cortex. In addition, the ACC can be reliably recorded with good test-retest reliability not only from listeners with normal hearing but also from listeners with hearing loss, HAs, and CIs. The ACC response can also be reliably recorded from infants and other children because it can be obtained even in the absence of attention. Moreover, it requires relatively few stimulus presentations to record a response with a good signal-to-noise ratio. Most importantly, it shows rea- 
sonable agreement with behavioral measures. These factors are positive for the potential clinical application of the ACC.

Overall, these findings suggest that the ACC may be a promising tool for the objective clinical evaluation of auditory discrimination and/or speech perception capacity. In the future, information obtained using the ACC might lead to improved counseling, rehabilitation, and outcomes.

\section{REFERENCES}

1) Hillyard SA, Picton TW. On and off components in the auditory evoked potential. Percept Psychophys 1978;24:391-8.

2) Martin BA, Boothroyd A. Cortical, auditory, event-related potentials in response to periodic and aperiodic stimuli with the same spectral envelope. Ear Hear 1999;20:33-44.

3) Whiting KA, Martin BA, Stapells DR. The effects of broadband noise masking on cortical event-related potentials to speech sounds /ba/ and /da/. Ear Hear 1998;19:218-31.

4) Näätänen R, Gaillard AW, Mäntysalo S. Early selective-attention effect on evoked potential reinterpreted. Acta Psychol (Amst) 1978; 42:313-29.

5) Picton TW. The neurophysiological evaluation of auditory discrimination. Ear Hear 1995;16:1-5.

6) Sutton S, Braren M, Zubin J, John ER. Evoked-potential correlates of stimulus uncertainty. Science 1965;150:1187-8.

7) Martin BA, Tremblay KL, Korczak P. Speech evoked potentials: from the laboratory to the clinic. Ear Hear 2008;29:285-313.

8) Ostroff JM, Martin BA, Boothroyd A. Cortical evoked response to acoustic change within a syllable. Ear Hear 1998;19:290-7.

9) Martin BA, Boothroyd A. Cortical, auditory, evoked potentials in response to changes of spectrum and amplitude. J Acoust Soc Am 2000;107:2155-61.

10) Michalewski HJ, Starr A, Nguyen TT, Kong YY, Zeng FG. Auditory temporal processes in normal-hearing individuals and in patients with auditory neuropathy. Clin Neurophysiol 2005;116:669-80.

11) Pratt H, Starr A, Michalewski HJ, Bleich N, Mittelman N. The N1 complex to gaps in noise: effects of preceding noise duration and intensity. Clin Neurophysiol 2007;118:1078-87.

12) Lister JJ, Maxfield ND, Pitt GJ. Cortical evoked response to gaps in noise: within-channel and across-channel conditions. Ear Hear 2007;28:862-78.

13) Atcherson SR, Gould HJ, Mendel MI, Ethington CA. Auditory N1 component to gaps in continuous narrowband noises. Ear Hear 2009; 30:687-95.

14) Harris KC, Mills JH, Dubno JR. Electrophysiologic correlates of intensity discrimination in cortical evoked potentials of younger and older adults. Hear Res 2007;228:58-68.

15) Dimitrijevic A, Lolli B, Michalewski HJ, Pratt H, Zeng FG, Starr A. Intensity changes in a continuous tone: auditory cortical potentials comparison with frequency changes. Clin Neurophysiol 2009; 120:374-83.

16) Dimitrijevic A, Starr A, Bhatt S, Michalewski HJ, Zeng FG, Pratt H. Auditory cortical N100 in pre- and post-synaptic auditory neuropathy to frequency or intensity changes of continuous tones. Clin Neu- rophysiol 2011;122:594-604.

17) Harris KC, Mills JH, He NJ, Dubno JR. Age-related differences in sensitivity to small changes in frequency assessed with cortical evoked potentials. Hear Res 2008;243:47-56.

18) Tremblay KL, Friesen L, Martin BA, Wright R. Test-retest reliability of cortical evoked potentials using naturally produced speech sounds. Ear Hear 2003;24:225-32.

19) Tremblay KL, Billings CJ, Friesen LM, Souza PE. Neural representation of amplified speech sounds. Ear Hear 2006;27:93-103.

20) Friesen LM, Tremblay KL. Acoustic change complexes recorded in adult cochlear implant listeners. Ear Hear 2006;27:678-85.

21) Martinez AS, Eisenberg LS, Boothroyd A. The Acoustic Change Complex in Young Children with Hearing Loss: A Preliminary Study. Semin Hear 2013;34:278-87.

22) Small SA, Werker JF. Does the ACC have potential as an index of early speech discrimination ability? A preliminary study in 4-monthold infants with normal hearing. Ear Hear 2012;33:e59-69.

23) Martin BA. Can the acoustic change complex be recorded in an individual with a cochlear implant? Separating neural responses from cochlear implant artifact. J Am Acad Audiol 2007;18:126-40.

24) Brown CJ, Etler C, He S, O’Brien S, Erenberg S, Kim JR, et al. The electrically evoked auditory change complex: preliminary results from nucleus cochlear implant users. Ear Hear 2008;29:704-17.

25) Kim JR, Brown CJ, Abbas PJ, Etler CP, O'Brien S. The effect of changes in stimulus level on electrically evoked cortical auditory potentials. Ear Hear 2009;30:320-9.

26) He S, Grose JH, Buchman CA. Auditory discrimination: the relationship between psychophysical and electrophysiological measures. Int J Audiol 2012;51:771-82.

27) He S, Grose JH, Teagle HF, Woodard J, Park LR, Hatch DR, et al. Acoustically evoked auditory change complex in children with auditory neuropathy spectrum disorder: a potential objective tool for identifying cochlear implant candidates. Ear Hear 2015;36:289-301.

28) Starr A, Picton TW, Sininger Y, Hood LJ, Berlin CI. Auditory neuropathy. Brain 1996;119(Pt 3):741-53.

29) Zeng FG, Oba S, Garde S, Sininger Y, Starr A. Temporal and speech processing deficits in auditory neuropathy. Neuroreport 1999;10: 3429-35.

30) Won JH, Clinard CG, Kwon S, Dasika VK, Nie K, Drennan WR, et al. Relationship between behavioral and physiological spectral-ripple discrimination. J Assoc Res Otolaryngol 2011;12:375-93.

31) Henry BA, Turner CW, Behrens A. Spectral peak resolution and speech recognition in quiet: normal hearing, hearing impaired, and cochlear implant listeners. J Acoust Soc Am 2005;118:1111-21.

32) Litvak LM, Spahr AJ, Saoji AA, Fridman GY. Relationship between perception of spectral ripple and speech recognition in cochlear implant and vocoder listeners. J Acoust Soc Am 2007;122: 982-91.

33) He S, Grose JH, Teagle HF, Woodard J, Park LR, Hatch DR, et al. Gap detection measured with electrically evoked auditory eventrelated potentials and speech-perception abilities in children with auditory neuropathy spectrum disorder. Ear Hear 2013;34:733-44.

34) He S, Grose JH, Teagle HF, Buchman CA. Objective measures of electrode discrimination with electrically evoked auditory change complex and speech-perception abilities in children with auditory neuropathy spectrum disorder. Ear Hear 2014;35:e63-74. 